\title{
Corrigendum to "Waterflooding Technique to the Kashkari Oilfield in the North Part of Afghanistan"
}

\author{
Zabihullah Mahdi,*, Kazunori Abe, Khwaja Naweed Seddiqi, Hikari Fujii \\ Graduate School of International Resource Sciences, Akita University Department of Earth Resource \\ Engineering and Environmental Science 1-1 Tegata gakuen-machi, Akita 010-8502, Japan \\ *Corresponding author.Email: zabih.mahdi@gmail.com
}

In the original paper, the Acknowledgments section is missing. The completed Acknowledgments section appears below. The authors are sorry for any inconveniences caused by this error.

DOI of original article: https://doi.org/10.2991/ahcps.k.211004.018

\section{ACKNOWLEDGMENTS}

Authors would like to express their gratitude to Mr. Ahmad Rateb Popal and they are deeply grateful to Watan Oil and Gas Group for permission to have access and use row data of Amu Darya basin. We are particularly grateful for the assistance given by prof. Shigeo Honma. In addition, we would like to express our thanks to Akita University Support for Fostering Research Project, and JSPS KAKENHI Grant Number JP21K04959 for their valuable funding sources for doing this research. 